\title{
Exploration of Digital Twin Design Mechanism of the Deep in Situ Rock Insulation Coring Device
}

\author{
Bo Yu ${ }^{(D)}{ }^{1}$ Heping Xie, ${ }^{1,2}$ Ling Chen $\mathbb{D}^{\circ},{ }^{1}$ Wu Zhao, ${ }^{1}$ and Zhiqiang He $\mathrm{He}^{1,2}$ \\ ${ }^{1}$ School of Mechanical Engineering, State Key Laboratory of Hydraulics and Mountain River Engineering, College of Water Resource \\ and Hydropower, Sichuan University, Chengdu 610065, China \\ ${ }^{2}$ Guangdong Provincial Key Laboratory of Deep Earth Sciences and Geothermal Energy Exploitation and Utilization, Institute of \\ Deep Earth Sciences and Green Energy, College of Civil and Transportation Engineering, Shenzhen University, \\ Shenzhen 518061, China \\ Correspondence should be addressed to Ling Chen; chenlingscu@scu.edu.cn
}

Received 14 August 2020; Revised 20 August 2020; Accepted 5 September 2020; Published 22 September 2020

Academic Editor: Zhijie Wen

Copyright (C) 2020 Bo Yu et al. This is an open access article distributed under the Creative Commons Attribution License, which permits unrestricted use, distribution, and reproduction in any medium, provided the original work is properly cited.

\begin{abstract}
With the development of the resource exploration and environmental science drilling, strict and scientific requirements are put forward for the samples taken from drilling. It is significant to keep the original appearance of the core and obtain the in situ core for the analysis of deep geological fluid and the exploration of the law of geological disasters caused by large-scale geological exploitation. To achieve the high-fidelity in situ core of deep rock, the development of the corresponding deep in situ fidelity coring device should involve the insulation coring device. The development of deep in situ fidelity coring device is a typical sophisticated product design. There are many problems in the design process, such as multimodules, multidisciplinary, crossdomain, and high coupling, which makes it more difficult for users to participate in product design and understand the product design intention. Digital twin technology, such as time data collection, accelerated iterative optimization, and highfidelity rendering, provides users with an immersive experience and deepens their understanding of the product design intention. The exploration of the novel design model combined digital twin technology with innovative design theory. Digital twin innovative design of the deep in situ insulation coring device is based on the innovative design method, which built a digital connection between the pre-research test platform and the corresponding simulation models. This digital twin to help users participate in product design and understand the product design process. Finally, the TOPSIS evaluation model was used to calculate the user's score on the design scheme, which increased by $27.64 \%$, which improves the overall efficiency of product design. This paper provides a practical design method and technical means for the design of the deep in situ insulation coring device based on the geological mechanism and control theory of thermal insulation core.
\end{abstract}

\section{Introduction}

Drilling sampling technology plays an irreplaceable role in today's major engineering neighborhoods, from the geological exploration to the oil and gas resource assessment which cannot be separated from sampling technology $[1,2]$. Nowadays, with the continuous development and progress of drilling sampling technology, scientific research workers have put forward higher and higher requirements for the quality of the samples taken out. Obtaining high-fidelity core is of great significance for the formation of corresponding deep mining theory, but the current coring technology lacks mea- sures to maintain in situ temperature and pressure. The temperature is related to the gas adsorption constant of coal samples. It is generally believed that the adsorption capacity of coal to gas decreases with the increase of temperature; with the increase of reservoir temperature, the thermal movement of gas molecules accelerates, and the adsorption capacity of shale gas weakens $[3,4]$.

The lack of in situ temperature maintenance will lead to distortion in the evaluation of fluid resources such as shale gas and coal mine gas. On the other hand, large-scale exploitation may lead to submarine geological landslide and other disasters, which is one of the technical bottlenecks restricting 


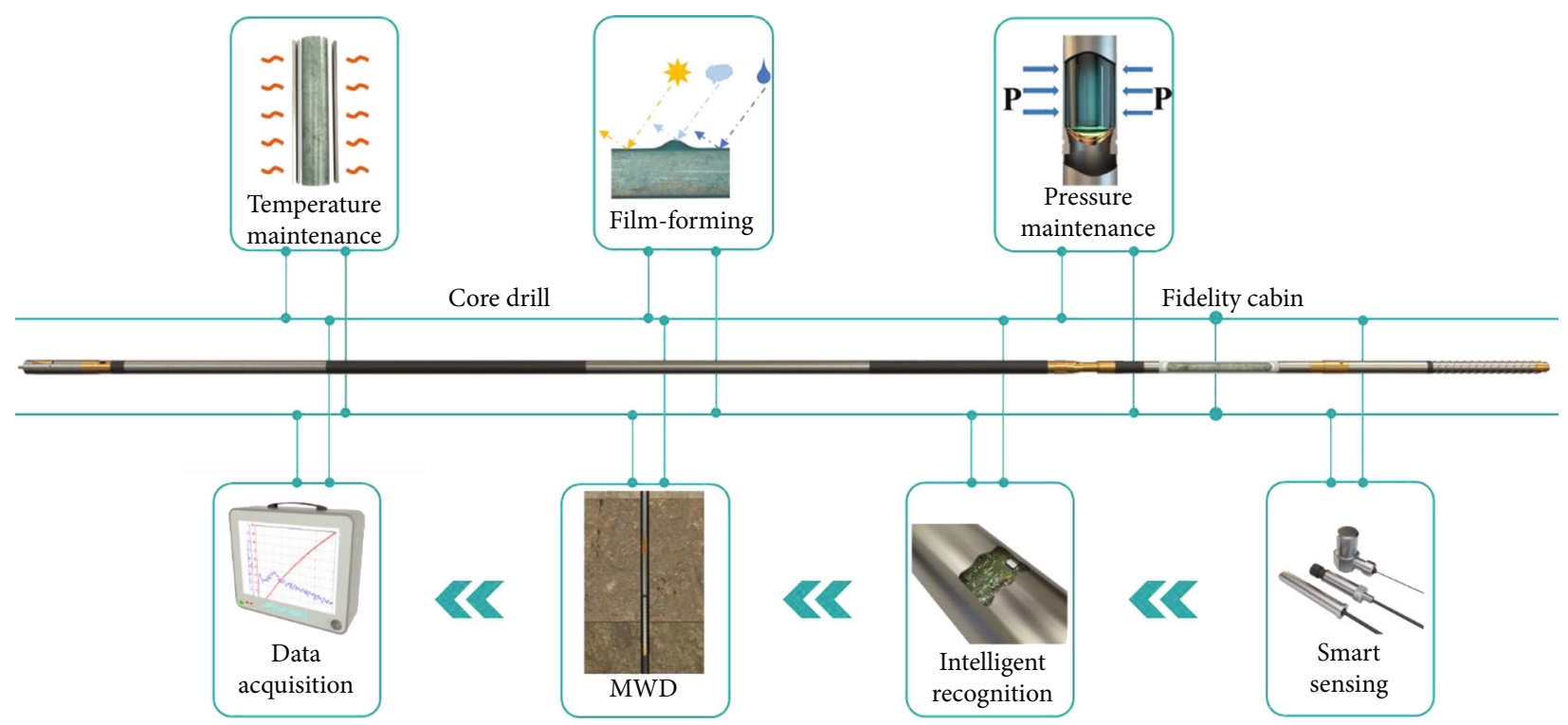

Figure 1: Module composition of the deep in situ fidelity coring system.

the commercial exploitation of natural gas hydrate. Deep high-fidelity coring technology can provide methods and means for the deep-sea geological exploration and deep-sea storage resources evaluation, and it is significant to find out the deep-sea geological structure and ensure safe mining. How to realize deep high-fidelity coring technology has become a theoretical and technical problem that needs to be solved urgently [5-8].

Therefore, it is necessary to research and develop the principle, technology, and equipment of high-fidelity in situ coring tools. The traditional drilling coring technology lost in situ information of deep rock core. Professor Xie proposed the coring method of "Five Guarantees" (pressure keeping, heat preservation, quality preservation, moisture preservation, and light preservation) and develop and integrate high-quality coring technology based on traditional drilling coring equipment fidelity technology [6]. The high-fidelity core obtained by "Five Guarantees" coring technology aims to keep the parameters of core in situ occurrence environment unchanged during drilling, operation, and storage.

The in situ fidelity coring device is mainly composed of three parts: intelligent identification module, "Five Guarantees" control module, and MWD module $[9,10]$. It belongs to the typical complex product design. In the conceptual design stage, modular R \& D can be used to decompose the design space and to decompose the complex design problems into several relatively simple design problems. Each functional module is handed over to the corresponding professional team for research and development, respectively. The parallel R \& D not only ensure the professionalism of R \& $\mathrm{D}$ but also improves the efficiency and efficiency of $\mathrm{R} \& \mathrm{D}$. Finally; the modules are spliced and decoupled to integrate the complex product system [8]. The module composition of the deep in situ fidelity coring system is shown in Figure 1.

The deep in situ fidelity coring device is a typical complex design product, which has a complex structure, intensive technology, and high manufacturing requirements and costs.
During the design process, there is the complex process management and changeable customer requirements, which usually involve multidisciplinary fields such as machinery, communication, electronics, hydraulic, pneumatic, and software. Each component and subsystem may be composed of parts from different professional fields. These tens of thousands of components and subsystems need direct or indirect interaction [11-13], which increases the complexity and difficulty of the complex product design optimization [14]. To design complex products, designers need to master the characteristics of various disciplines and have very comprehensive professional knowledge and design experience.

The process of the complex product design is divided into four stages: clear task, conceptual design, basic design, and detailed design. Among them, the conceptual design is the key to determine whether the designed product can meet the product performance objectives, which covers the product selection design, scheme design, scheme design review, and other stages. According to statistics, 75\% of the product quality problems are generated in the product development and design stage. In the design stage, the more perfect the scheme is, the less associated problems will be caused, thus reducing the cost, shortening the development cycle, and improving the competitiveness of products [15].

Consideration of digital twin technology into product design, the design problems and contradictions encountered in the design of fidelity device are effectively solved. Moreover, the iterative optimization of conceptual design is accelerated, and the high-fidelity design scheme is presented, which provides new design ideas and methods for the fidelity device.

\section{Virtual Design and Digital Twin Technology in Product Design}

Virtual design is based on the "virtual reality" technology, taking mechanical products as the object to design in the 
virtual environment. It makes full use of the simulation technology to design, manufacture, and test the virtual digital model on the computer and find the possible problems in various aspects [16]. At present, domestic and foreign scholars have done a lot of research on the solution process of the complex product conceptual design combined with virtual design technology and achieved certain results. The University of Wisconsin Madison in the United States has developed a virtual conceptual design system COVIRDS, which uses gesture recognition and gesture tracking system to enable users to interact with the virtual modeling environment more intuitively [17]; B. Jung of Bielefeld University in Germany built a virtual assembly system based on the concept of construction tools; The CIM Research Institute of Shanghai Jiaotong University puts forward the 5-tier structure of virtual manufacturing technology and studies some key technologies of implementing virtual manufacturing technology. The computer department of Beijing University of Aeronautics and Astronautics has carried out some research on VR basic knowledge [18] and focuses on the representation and processing of physical characteristics in the virtual environment. The CIMS Engineering Research Center of Tsinghua University has proposed a virtual manufacturing architecture, which is a virtual manufacturing, virtual production, and virtual enterprise framework based on the product data management integration.

Digital twin technology first appeared in the United States during the cold war. In order to ensure the safety of astronauts, before launching the spacecraft, the spacecraft twins were established on the ground for experiments and data collection, but the concept of digital twins was not yet available [19]. The concept of "digital twins" first appeared in the course of Professor Grives of the University of Michigan and was defined as a three-dimensional model, including physical products of physical space, digital twins of virtual space, and data and interface of information interaction between physical and virtual space [20].

In terms of product design, Dassault has established a 3D experience platform based on digital twin for the innovative design of complex products. The product design model in the information world is continuously improved by using the information feedback from users and feedback to the physical entity product for improvement [12]. In foreign countries, Victor et al. gave the mathematical definition of DT in product design for design decision [21]; Schleich et al. proposed a comprehensive reference model based on the shape of the surface model based on DT, as DT of physical products in the design and manufacturing stage [22]; At home, Zhuang et al. analyzed the implementation possibility of DT in the product design stage and considered that the product definition based on the model is an important means to construct the product DT [23]; Liu et al. proposed the intelligent scheme design framework by using DT and digital ties according to the requirements of intelligent development and development of spacecraft; Tao et al. made a preliminary study on the DT design framework [24]; Hao et al. made the connotation of the complex product design based on DT which is analyzed and the ring design framework of complex product based on DT which is proposed [25].
To sum up, domestic and foreign scholars have developed theoretical research foundation on the conceptual design of complex products, but few in practical application. The rationality of the design scheme largely depends on the experience of designers and the operation site. Even in the face of the same design task, the details of different designers' designs are often quite different. For the complex, large-scale equipment, the design process involved in a too wide field, many components, complex motion mechanism, strict functional requirements, and other factors. Designers have to carry out functional disassembly and modular design for the design object and build a preresearch test platform through the laboratory in the conceptual design stage to form a set of boundary conditions. After a reasonable simplification of the principle of the experimental model to replace the actual working condition of the product, the functional verification of the product is carried out to ensure the rationality of the conceptual product design. On the one hand, this design method follows the traditional mechanical product design process, belonging to serial execution, with many iterations and low design efficiency. On the other hand, the principle model used for a preresearch experiment is quite different from the object in the actual working condition from the intuitive appearance. How to design an effective preresearch experiment according to the user's needs and how to make the user fully understand the one-to-one correspondence between the preresearch experiment model and the final actual object are the key to determine the reliability of the complex product conceptual design.

\section{The Process Model of the Digital Twin Conceptual Design}

Product design based on digital twin refers to that driven by the product twin data. The data connection was existing physical experimental products and corresponding virtual twins in the model. The interactive synchronous iteration technology is used to accelerate the convergence iteration of product design. It is also continuously new, unique, and valuable product concepts, transforms them into reliable product design schemes, and reduces the inconsistency of the products between the actual behavior and the expected behavior of the design. At the same time, the virtual and hyperrealistic multidimensional high-fidelity characteristics of digital twin technology can reflect the real-time performance parameters of products, which significantly improves the representation authenticity of physical entities [14, 15]. The combination of digital twin technology and the product innovation conceptual design is helpful for designers to carry out the rapid and effective conceptual design of complex products, convey product design information to users, and reconstruct users' virtual experience about design objects. It is also helpful for users to understand the designer's design intent and put forward a more effective conceptual design process model for complex products, and the design requirements can increase the effectiveness and importance of user's participation in product design. Digital twin technology plays an essential role in the rapid and effective convergence of product design 


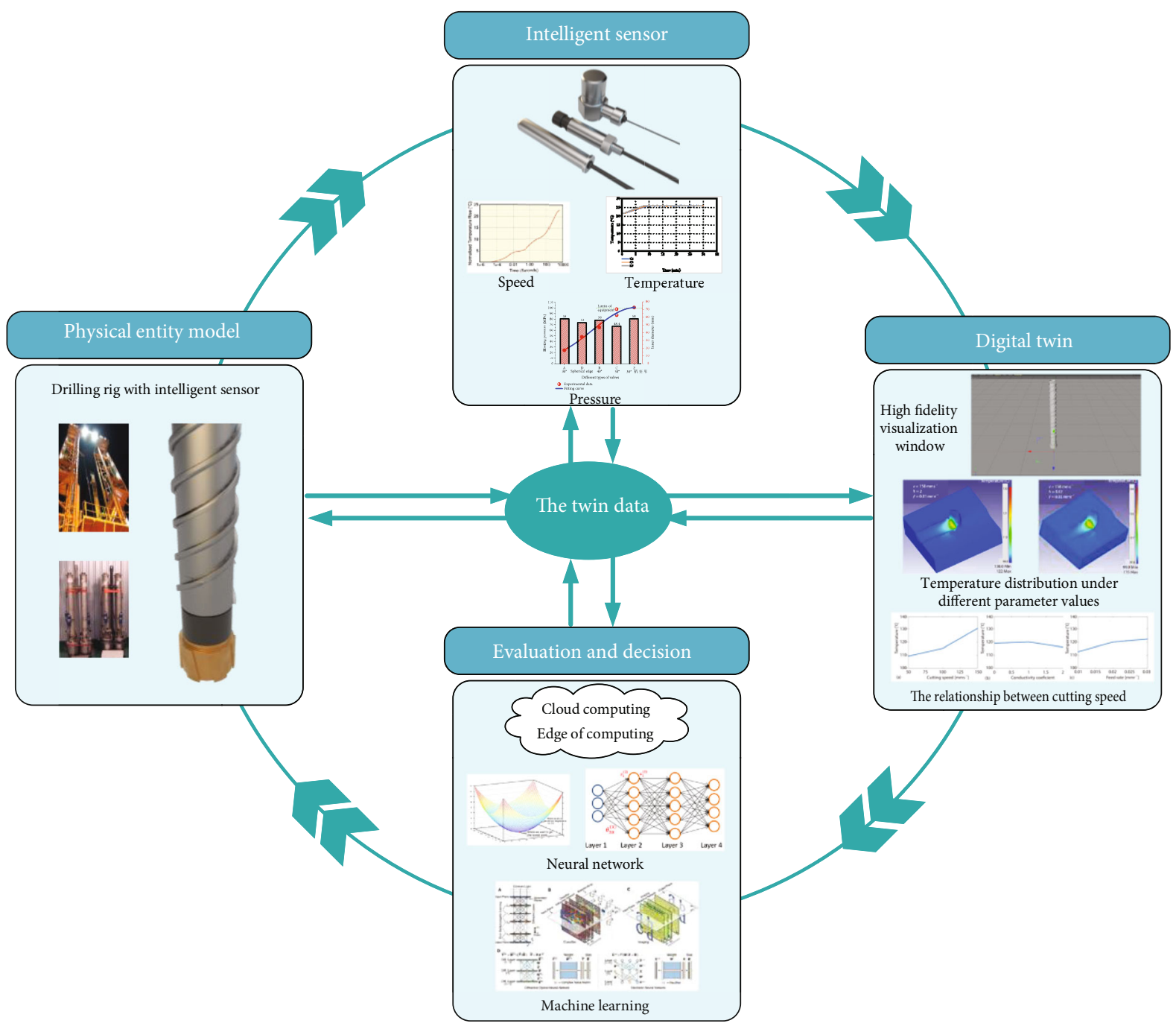

FIGURE 2: Schematic diagram of the digital twin model.

and the information consistency between users and designers in the conceptual design stage of complex products.

According to the critical elements of the digital twin, a complete digital twin system is preliminarily divided into five modules: physical object, intelligent sensor, digital twin, evaluation decision, and twin data. The intelligent sensor is used as the data connection channel to transmit the design and experimental data generated in the preliminary research experiment of the physical entity model to the corresponding digital twin in real-time. Through the real-time simulation, optimization and final evaluation, and decision-making system in the digital twin, the model data after iterative convergence is fed back to the design and experiment of the physical entity model, so as to achieve the fast and effective updating iteration of the experimental model. The digital twin model is shown in Figure 2 [26].

Taking the design of the temperature fidelity system, one of the "Five Guarantees" in deep in situ fidelity coring technology as the object, a new conceptual design process model of complex products is constructed by combining digital twin technology. Through the ad axiomatic design theory, the pre- liminary requirements of the design object are obtained, and the design requirement parameter vector and design matrix are obtained. The coupling problems such as contradictions and conflicts in the design are solved by using the TRIZ innovative design method, and the preliminary conceptual design of the product is formed. According to the conclusion of the conceptual design, the corresponding laboratory-based preresearch test platform is built. At the same time, the corresponding digital twin is constructed, and the digital bridge is built. The effective iteration and optimization of the preresearch test platform are accelerated through numerical simulation, real-time interaction of result data, and high-fidelity real-time rendering, so as to obtain the optimal preresearch platform for conceptual design quickly. Then, through the high-fidelity real-time rendering digital model, users can fully understand the one-to-one correspondence between the preresearch test platform and the real insulation coring system and deepen the user's understanding of the designer's product design intention. Finally, the TOPSIS system evaluation model is used to calculate the user's score of conceptual design results before and after the use of digital twin 


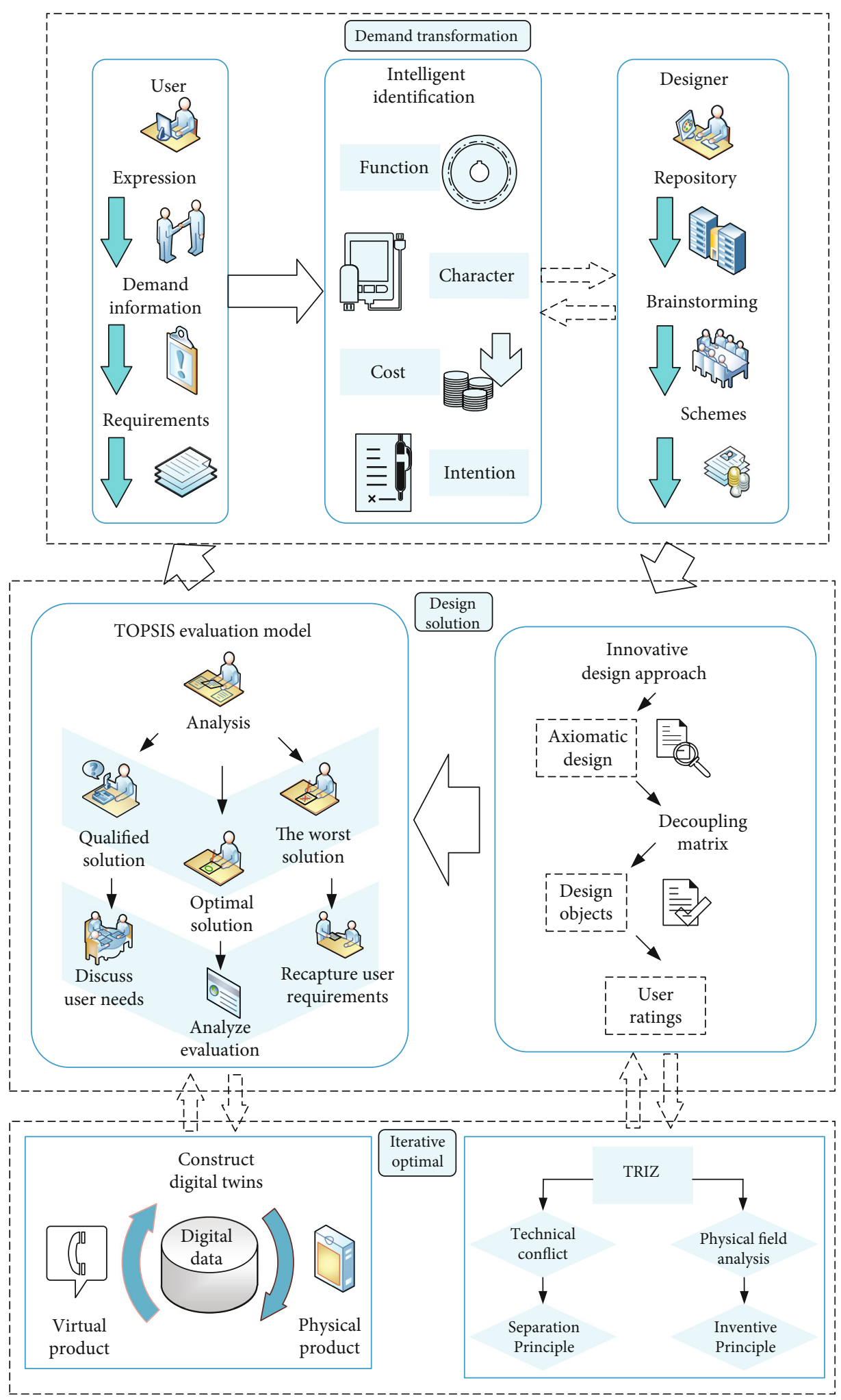

FIgure 3: The design process model based on digital twin Technology.

technology. The specific design process model is shown in Figure 3.

Axiomatic design theory (AD) is a top-down design process [27], which forms a mapping relationship between user domain, functional domain, and structural domain $[28,29]$.
Based on the design principle of $\mathrm{AD}$, the "requirement function" transformation process is the process of establishing user requirements and functional requirements.

TRIZ (Theory of Inventive Problem-Solving, the original language is Russian) was proposed by Genrich s. altshuller in 
1964 [30]. The theory is composed of 39 engineering parameters and contradiction matrix, 40 invention principles, and four separation principles and physical contradictions and considers that the essence of innovation and invention is the process of solving contradictions [31, 32].

\section{The Digital Twin Conceptual Design of Complex Equipment in Deep Engineering}

4.1. Requirement Acquisition and Function Transformation of the Design System. The active thermal insulation system used in the coring environment is different from the conventional active thermal insulation device. The extreme working conditions must be considered in the design of the whole device. The main consideration here is how to realize the active constant temperature control and rapid response of the fidelity device under the conditions of small space in the deep and low energy supply, as well as real-time data acquisition and display.

According to AD theoretical rules, the functional requirements of the deep in situ insulation system are described in Table 1.

The functional requirement vector of the deep active thermal insulation device can be established from Table 1, as shown in formula (1)

$$
\left[\begin{array}{c}
\mathrm{FR}_{1} \\
\mathrm{FR}_{2} \\
\mathrm{FR}_{3} \\
\mathrm{FR}_{4}
\end{array}\right]=\left[\begin{array}{c}
\text { Active cooling function } \\
\text { Active heating function } \\
\text { Small space work } \\
\text { Energy supply }
\end{array}\right] \text {. }
$$

4.2. Establish the Design Parameter Vector and Design Matrix. After solving the design parameter, the solution considered the application of current coring devices in active thermal insulation. The Peltier refrigerator is used as the active refrigeration device; for the device with the active temperature rising, there is no precedent in the field of deep coring at home and abroad, and the heating film or graphene is used as the design parameter $\mathrm{DP}_{2}$. For the deep small space operation, because the active thermal insulation device should be integrated into the whole coring device, the drilling rig shell structure is used as the design parameter $\mathrm{DP}_{3}$. The corresponding design parameters $\mathrm{DP}_{4}$ of the energy supply function are cables or batteries. The functional requirements are compared with the design parameters, as shown in Table 2.

To sum up, the relationship between the functional requirements and the design parameter satisfying the element of the design matrix is as follows.

$$
\left[\begin{array}{l}
\mathrm{FR}_{1} \\
\mathrm{FR}_{2} \\
\mathrm{FR}_{3} \\
\mathrm{FR}_{4}
\end{array}\right]=\left[\begin{array}{llll}
1 & 0 & 0 & 0 \\
0 & 1 & 0 & 0 \\
1 & 1 & 1 & 0 \\
0 & 0 & 0 & 1
\end{array}\right] \times\left[\begin{array}{c}
\mathrm{DP}_{1} \\
\mathrm{DP}_{2} \\
\mathrm{DP}_{3} \\
\mathrm{DP}_{4}
\end{array}\right]
$$

If the design matrix in formula (2) is a triangular matrix, decoupling design is needed.
TABLE 1: Comparison of user requirements and functional requirements.

\begin{tabular}{lc}
\hline User demand $\left\{\mathrm{CA}_{\mathrm{S}}\right\}$ & Functional requirement $\left\{\mathrm{FR}_{\mathrm{S}}\right\}$ \\
\hline $\begin{array}{l}\text { Cooling the core } \mathrm{CA}_{1} \\
\text { Heating the core } \mathrm{CA}_{2}\end{array}$ & Active cooling function $\mathrm{FR}_{1}$ \\
$\begin{array}{l}\text { It can work in deep and } \\
\text { narrow space } \mathrm{CA}_{3}\end{array}$ & Small space work $\mathrm{FR}_{3}$ \\
$\begin{array}{l}\text { It can realize long-distance } \\
\text { energy supply } \mathrm{CA}_{4}\end{array}$ & Energy supply $\mathrm{FR}_{4}$ \\
\hline
\end{tabular}

TABLE 2: Comparison table of functional requirements and design parameters.

\begin{tabular}{lc}
\hline Functional requirement $\left\{\mathrm{FR}_{\mathrm{S}}\right\}$ & Design parameters $\left\{\mathrm{DP}_{\mathrm{S}}\right\}$ \\
\hline Active cooling function $\mathrm{FR}_{1}$ & Peltier refrigeration unit $\mathrm{DP}_{1}$ \\
Active heating function $\mathrm{FR}_{2}$ & Graphene heating device $\mathrm{DP}_{2}$ \\
Small space work $\mathrm{FR}_{3}$ & Shell structure $\mathrm{DP}_{3}$ \\
Energy supply $\mathrm{FR}_{4}$ & Cable or battery $\mathrm{DP}_{4}$ \\
\hline
\end{tabular}

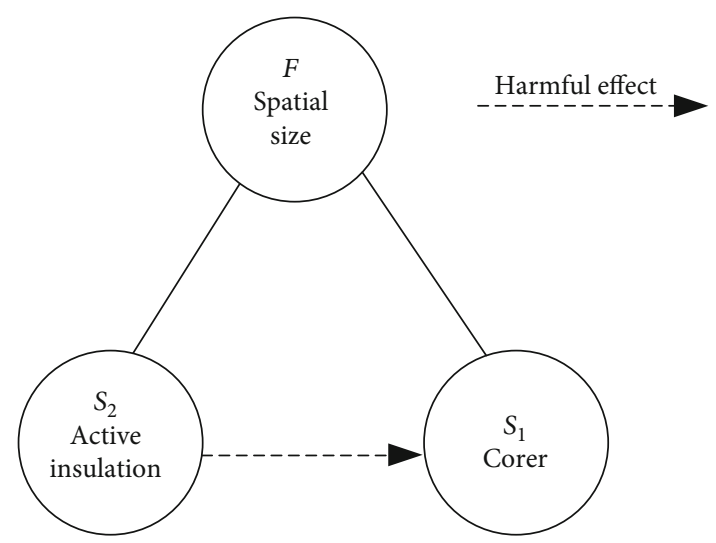

FIgURE 4: The material field model of the active insulation system.

4.3. The Decoupling Design Matrix with the Innovative Method. Design matrix $A$ is a matrix that needs to be decoupled. The design parameters of the Peltier refrigeration unit and graphene heating device will be greatly limited by the small space operation at the bottom hole. However, the transformation and expansion of the overall shell structure of the drilling rig do not conform to the process of the standardized module design. In this paper, the TRIZ innovative design method is used to transform design contradiction into TRIZ problem. Through material field analysis, separation principle and invention principle are used to find corresponding solutions $[33,34]$.

In the coupling problem of the current design, the material field model can be expressed as that the active heat preservation device (cooling and heating) is effective for the constant temperature of the coring device, but it is harmful in terms of space requirements. The establishment of the object field model is shown in Figure 4. $F$ is for space field, $S_{1}$ is for the coring device, and $S_{2}$ is the active insulation device. 
In TRIZ theory, the problem described by the matter field model analysis method is generally called the standard problem, which can be solved by 76 standard solutions. Among them, the method of eliminating harmful effects belongs to the second level of the first class of five types, that is, the destruction of the material field model. By using the first method of its five standard solutions, an effective solution can be put forward, that is, introducing a third substance to eliminate the harmful effects [35]. In this paper, liquid medium water is introduced between the active thermal insulation device and the constant temperature object coring device as the carrier of heat transfer. The heat is transferred to the coring device by circulating water, and the active thermal insulation device is not directly arranged outside the coring device, but the axial layout is adopted, which not only meets the active insulation function but also overcomes the limitation of deep space. The improved object field model is shown in Figure 5.

Aiming at the problems faced by the deep active thermal insulation function, the function of the deep active thermal insulation system is decomposed by the method of function description and definition. The active thermal insulation system is divided into five modules: constant temperature control, control system, energy supply, compact structure, and data display and acquisition. The active thermal insulation control system works under $12 \mathrm{~V}$ voltage. It adopts PID, control chip, and temperature sensor graphene and Peltier are used for active temperature control. Constant temperature control of the medium is realized under the condition of the micropump convection. Its functional decomposition is shown in Figure 6.

4.4. Setting up a Physical Preresearch Experimental Platform. According to the conceptual design results of the decoupling design matrix, the preresearch experimental platform is built. The main devices include $12 \mathrm{~V}$ low-voltage power supply, circulating minipump, circulating pipeline, graphene heater, Peltier cooler, temperature sensor, paperless recorder, and constant temperature experimental chamber. The schematic diagram of the system design and the physical diagram of the experimental platform is shown in Figures 7 and 8 , respectively.

4.5. Build the Digital Twin Preresearch Experimental Platform and High-Fidelity Model and Compare the User's Score. In order to make users fully understand the design intention of design products and the one-to-one correspondence between the conceptual design preresearch experimental platform and real products, the corresponding digital twin model was established. The digital twin model is described from four dimensions of geometry, physics, behavior, and rules, and the product function and structure are restored with high fidelity, and the optimized iterative design is realized. The corresponding relationship between the digital twin and the physical experiment platform is shown in Figure 9.

The experimental data of refrigeration and heating are collected by intelligent sensors in real time, and the data are transmitted to the digital twin model through the data transmission interface. Through the full element digital mapping

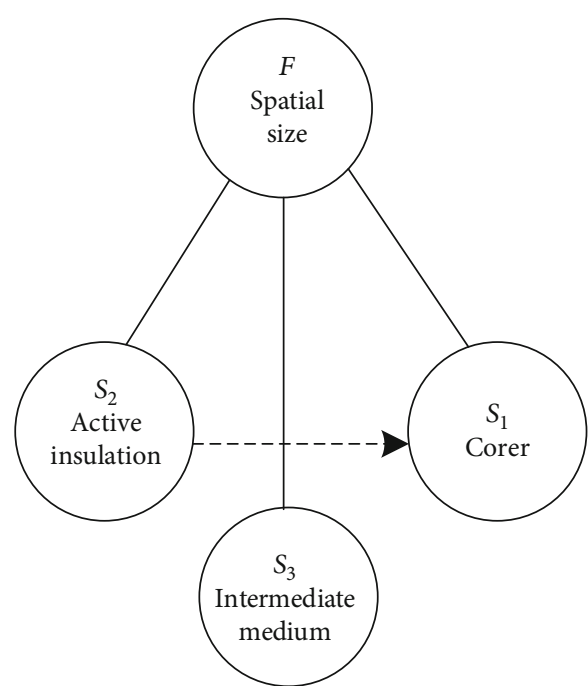

FIgURE 5: The improved material field model of the active heat preservation system.

of virtual product, the virtual real interaction feedback, monitoring, diagnosis, prediction, and control of the forming process, state, and behavior of the product entity are carried out. The digital simulation criteria, such as functions, are output for different dimensions of product design. It also makes decisions for the entity model and carries out product innovation and optimization iteration.

The test data collected by sensors arranged in the preresearch test platform, including circulating medium flow, graphene heater surface temperature, graphene physical size specification, heating container volume, and other data and information, are imported into the digital twin through the set data interface, and the working performance of the graphene heater is simulated and verified in the digital model. The working performance of the graphene heater is simulated by digital simulation, and the virtual verification is carried out in the digital model. The corresponding optimized design of the graphene heating device is carried out on the preresearch test platform. The test results are consistent with the design, which meets the design requirements and reduces the number and cycle of design test.

Figure 10 shows the construction of the digital twin of the preresearch experimental platform.

The temperature time curve of the measuring point generated by the graphene heating fluid medium calculated by digital simulation technology in digital twinning is compared with the curve of actual test, which is shown in Table 3. The coordinate of the temperature monitoring point is the center of the container, and the coordinate of the point is 0,0 . Since the medium used in the experiment is water, in order to ensure the safety and standardization of the experimental process, the temperature rise of the experimental and simulated water is not more than $100^{\circ} \mathrm{C}$.

Due to the complex and many factors in the real experiment, it is difficult to set the boundary conditions exactly in the numerical simulation, which leads to the deviation between the simulation results and the actual measurement results. But through the technical parameters and index 


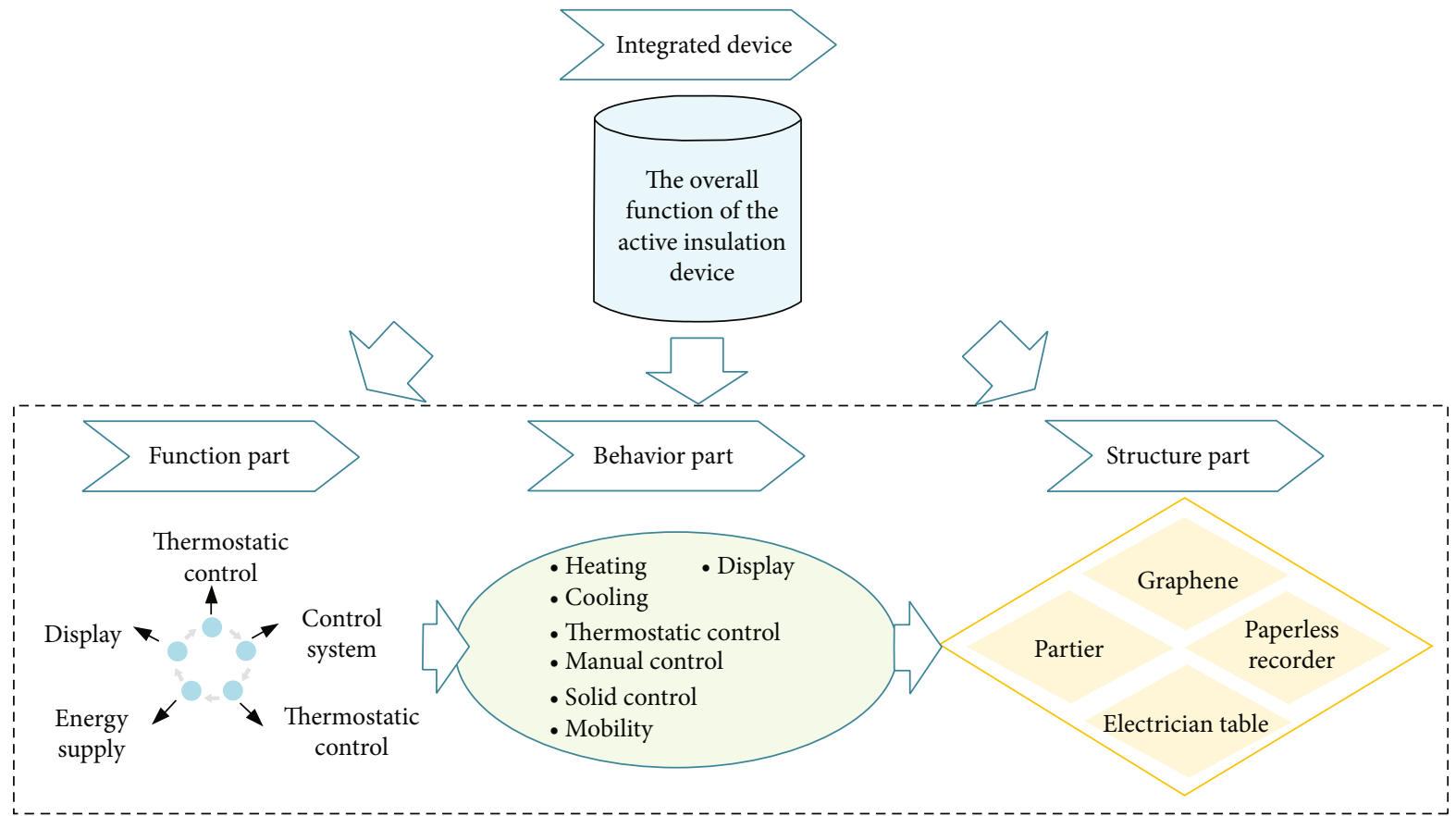

Figure 6: Functional decomposition of the deep active thermal insulation system.

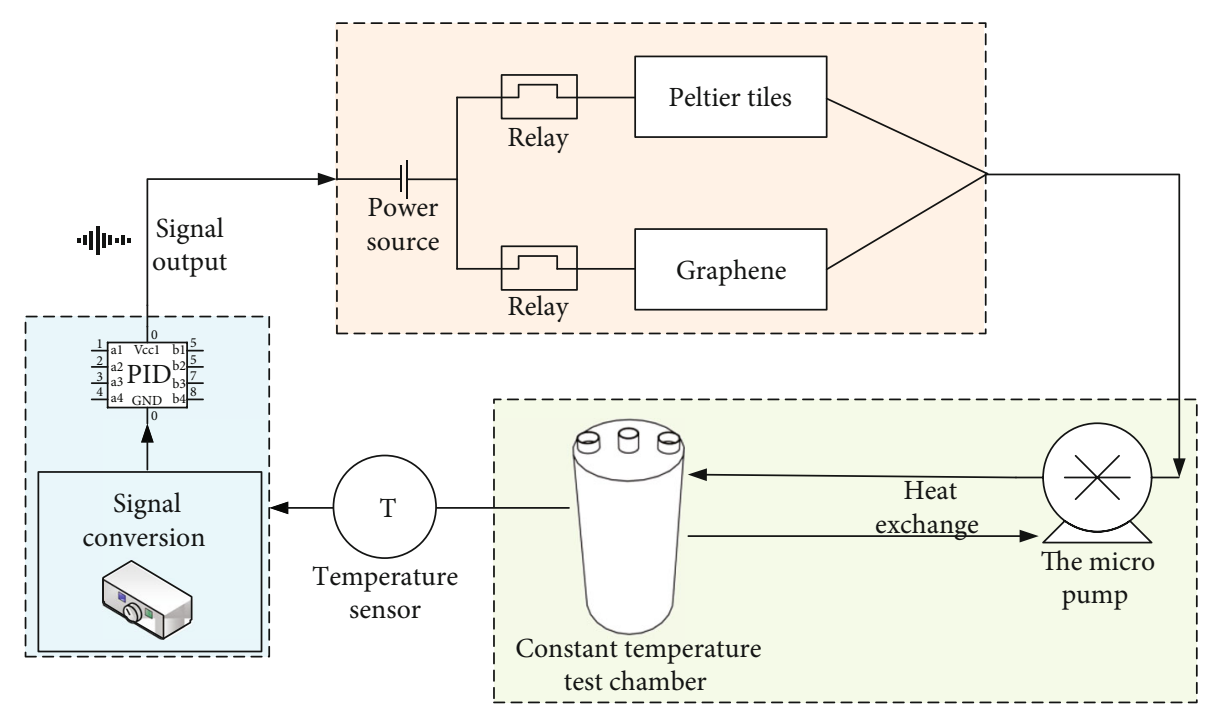

FiguRE 7: Schematic diagram of the insulation experiment system design.

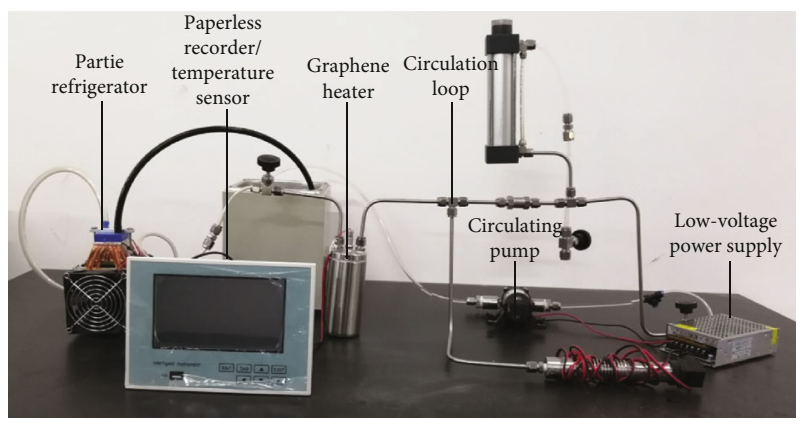

FIgURE 8: Setting up the experimental platform of the insulation experiment. curves in the digital twin of the preloading test platform, users have a clearer understanding and grasp of the one-toone correspondence between the principle test object and the final design object in the preresearch test and also more recognize the feasibility verification of the test.

After users fully understand and approve the preresearch experimental model in the conceptual design stage of complex products, the high-fidelity technology of the digital twin system can restore the final product design form to the greatest extent, provide users with realistic product sensory experience, and provide users with previrtual experience before the products are manufactured. The high-fidelity digital twin model of the deep in situ insulation system is shown in Figure 11. 


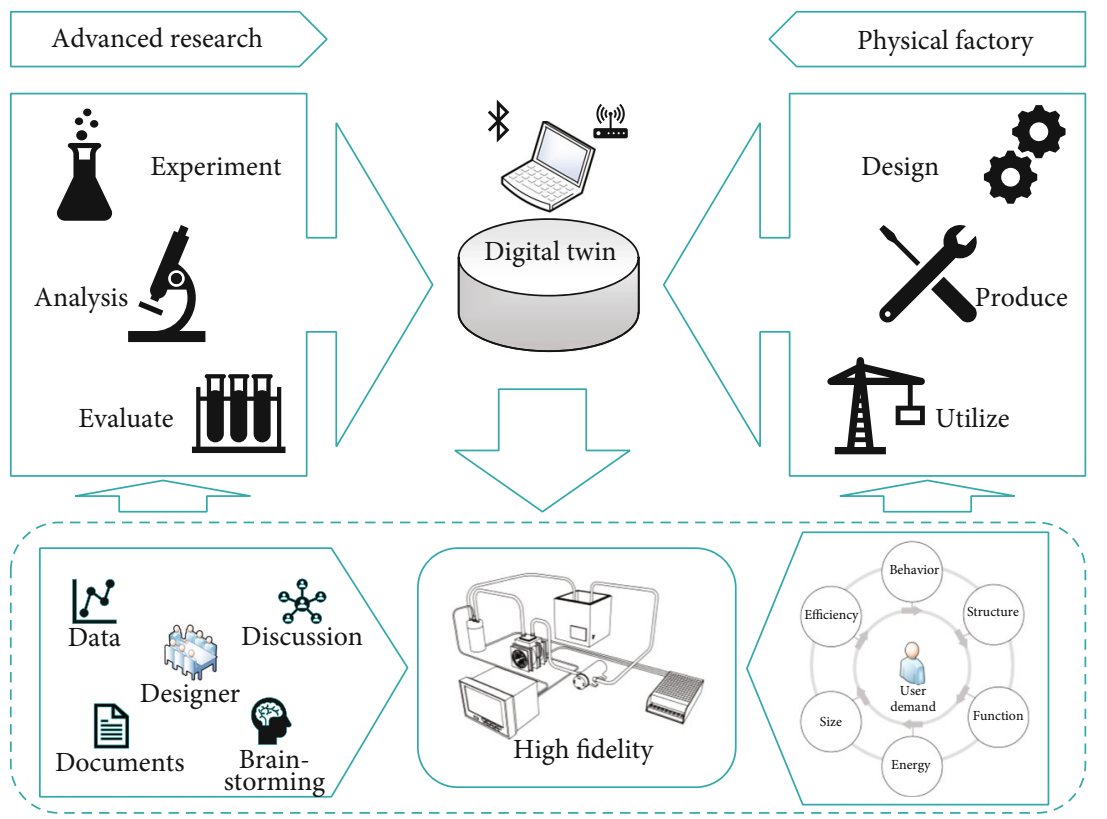

FIGURE 9: Correspondence between the digital model and real design object.

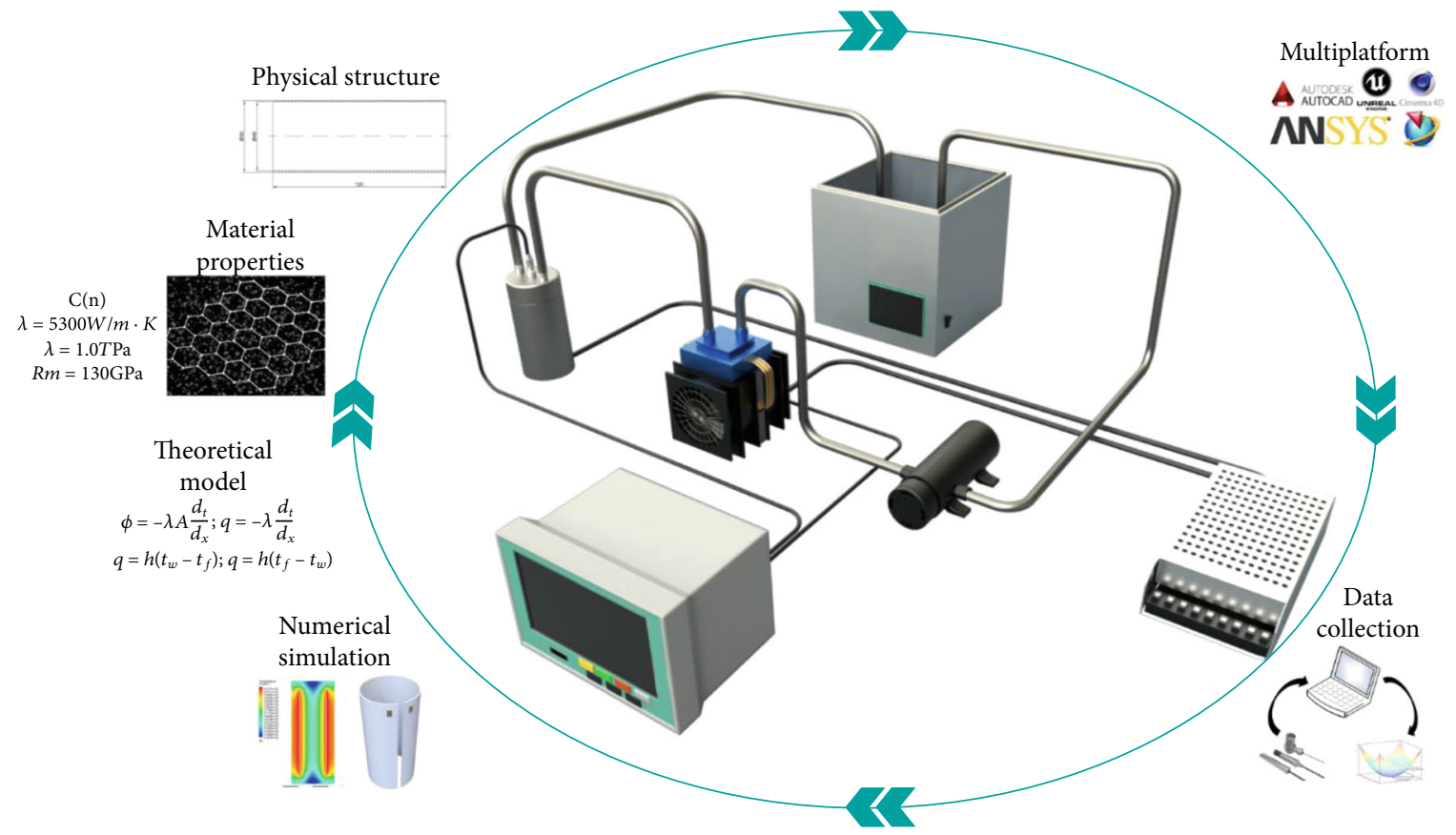

FIGURE 10: Corresponding digital twin object.

The TOPSIS method is suitable for the overall evaluation and benefit evaluation when the number of indicators and objects is small. The score of users on the initial preresearch experimental platform and the high-fidelity model after the establishment of digital twin were counted. The TOPSIS evaluation model was used to analyze the user's satisfaction with the main function requirements of the product. The distance between the score and the optimal solution is obtained by analyzing the user's satisfaction with the main function requirements of the product, which is used as the basis for evaluating the effectiveness of digital twin technology in the conceptual design stage of complex products.

According to the product function requirement, which can be used as the evaluation index of the design object, respectively, the score statistics of each index are shown in Table 4.

The traditional TOPSIS method, also known as ideal solution, is an effective method for multiobjective system optimization, ranking, evaluation, and decision-making. When the distance between a scheme and the ideal solution 
TABLE 3: Digital technology and test results contrast.

Digital simulation results

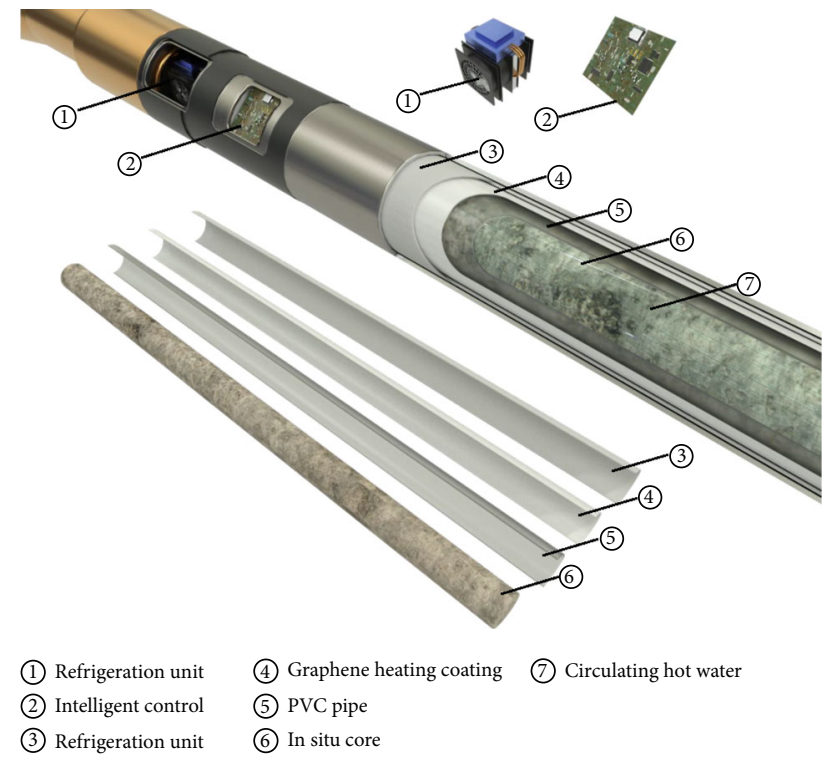

FIgURE 11: The high-fidelity digital twin model.

is the shortest, it can be considered as the best scheme $[36,37]$. The basic principle of the TOPSIS method is to rank the alternatives with the help of "ideal solution" and "negative ideal solution" of the multiobjective decisionmaking problem [38].

Firstly, the evaluation indexes were processed without quantification, that is, the original data were normalized:

$$
Z_{i j}=\frac{x_{i j}}{\sqrt{\sum_{i=1}^{4} x_{i j}^{2}}} .
$$

The $Z$ matrix without quantization is calculated as Table 5 .
TABLE 4: User rating table of functional requirements.

\begin{tabular}{lccc}
\hline $\begin{array}{l}\text { Serial } \\
\text { number }\end{array}$ & $\begin{array}{c}\text { Evaluating } \\
\text { indicator }\left(X_{i}\right)\end{array}$ & $\begin{array}{c}\text { Physical } \\
\text { preresearch } \\
\text { test platform }\end{array}$ & $\begin{array}{c}\text { Digital } \\
\text { twin }\end{array}$ \\
\hline 1 & Active cooling $\left(X_{1}\right)$ & 65.8 & 65.8 \\
2 & Active heating $\left(X_{2}\right)$ & 70.4 & 74.6 \\
3 & Small space work $\left(X_{3}\right)$ & 55.2 & 70.5 \\
4 & Energy supply $\left(X_{4}\right)$ & 68.6 & 62.9 \\
\hline
\end{tabular}

TABle 5: $Z$ matrix of the conversion index value.

\begin{tabular}{lccc}
\hline $\begin{array}{l}\text { Serial } \\
\text { number }\end{array}$ & $\begin{array}{c}\text { Evaluating } \\
\text { indicator }\left(Z_{i}\right)\end{array}$ & $\begin{array}{c}\text { Physical } \\
\text { preresearch } \\
\text { test platform }\end{array}$ & Digital twin \\
\hline 1 & Active cooling $\left(Z_{1}\right)$ & 0.504088037 & 0.479622158 \\
2 & Active heating $\left(Z_{2}\right)$ & 0.539328234 & 0.543766155 \\
3 & Small space work $\left(Z_{3}\right)$ & 0.422882365 & 0.513880884 \\
4 & Energy supply $\left(Z_{4}\right)$ & 0.525538592 & 0.458483796 \\
\hline
\end{tabular}

The optimal solution consists of the maximum value of each row in $Z$ :

$Z^{+}=(0.504088037,0.543766155,0.513880884,0.525538592)$

The worst case consists of the minimum value of each row in $Z$ :

$Z^{-}=(0.479622158,0.539328234,0.422882365,0.458483796)$. 
TABLE 6: $C$ values and sorting.

\begin{tabular}{lccc}
\hline $\begin{array}{l}\text { Serial } \\
\text { number }\end{array}$ & $\begin{array}{c}\text { Evaluating } \\
\text { indicator }\end{array}$ & $\begin{array}{c}\text { Physical preresearch } \\
\text { test platform }\end{array}$ & Digital twin \\
\hline 1 & $D^{+}$ & 0.091106671 & 0.071378742 \\
2 & $D^{-}$ & 0.071378742 & 0.091106671 \\
3 & $C_{i}$ & 0.43929323 & 0.560706769 \\
4 & Sorting results & 2 & 1 \\
\hline
\end{tabular}

The distance between each evaluation object and the best and worst plan was calculated

$$
D_{i}^{+}=\sqrt{\sum_{i=1}^{4}\left(\max Z_{i j}-Z_{i j}\right)^{2}} ; D_{i}^{-}=\sqrt{\sum_{i=1}^{4}\left(\min Z_{i j}-Z_{i j}\right)^{2}} .
$$

The results are as follows:

$$
\begin{aligned}
& D_{1}^{+}=0.091106671 ; D_{1}^{-}=0.071378742 . \\
& D_{2}^{-}=0.071378742 ; D_{2}^{-}=0.091106671
\end{aligned}
$$

Calculate the proximity degree $C_{i}$ between each evaluation object and the optimal scheme. The evaluation results of the design scheme can be given.

$$
\begin{aligned}
C_{i} & =\frac{D_{i}^{-}}{D_{i}^{+}+D_{i}^{-}} . \\
0 & \leq C_{i} \leq 1
\end{aligned}
$$

The larger $C_{i}$ is, the better the evaluation object is

$$
C_{1}=0.43929323 ; C_{2}=0.560706769 \text {. }
$$

The $C$ value and sorting table of the design scheme are shown in Table 6.

Through the TOPSIS model, the user evaluation and analysis of the preresearch test are carried out. From the ranking results, it can be seen that the conceptual design of complex products has been recognized by users to a higher degree through the high-fidelity restoration performance of digital twin technology.

\section{Conclusion}

Because of the lack of rock samples in the real situ environment in deep engineering theory, this paper studies the innovative design method of rock insulation coring equipment. It has laid a theoretical foundation for the development of scientific instruments for the study of geological mechanism. In the conceptual design stage of complex products, the process of module design, simplification of design elements, and preresearch test lead to the problems of the long design cycle, ill-conditioned design, and user's understanding deviation of the simplified preresearch test. In the conceptual design of the deep in situ insulation system, digital twin technology is introduced to generate the more intuitive and high-fidelity digital model. The comparison and optimization of the collected real-time test data and simulation results also accelerate the convergence of the overall product design process. Through the TOPSIS evaluation system, the user's recognition of the optimization of design is increased by $27.64 \%$ based on the original score. It can be seen in the application of digital twin technology in the conceptual design stage of complex products. The proposed model can help users understand the product design intention and information and participate in the conceptual design of complex products and to improve the overall effectiveness of the equipment design performance and design efficiency. However, due to the characteristics of the modular design of the sophisticated product design, how to organically associate and reorganize the separate design modules in the digital twin, solve the complex coupling problem between the modules, and deal with the multiple interaction and fusion of data and models in physical space and virtual space are all problems to be further studied.

\section{Data Availability}

The data used to support the findings of this study cannot be shared.

\section{Conflicts of Interest}

The authors declare that they have no conflicts of interest.

\section{Acknowledgments}

This work was supported by the National Natural Science Foundation of China (grant numbers 51827901 and 51822403) and the National Key Research and Development Project, China (Grant No. 2018YFB1700700). The financial aids are gratefully acknowledged.

\section{References}

[1] E. X. Zhao, M. L. Jia, J. P. Cai, and J. Y. Zhang, "Progress in fine, in situ, fidelity sampling techniques," Exploration Engineering (Rock \& Soil Drilling and Tunneling), vol. 32, no. 1, pp. 253-257, 2005.

[2] M. Z. Gao, R. Zhang, J. Xie, G. Y. Peng, B. Yu, and P. G. Ranjith, "Field experiments on fracture evolution and correlations between connectivity and abutment pressure under top coal caving conditions," International Journal of Rock Mechanics and Mining Sciences, vol. 111, no. 2018, pp. 84-93, 2018.

[3] C. Guan, S. Liu, C. Li, Y. Wang, and Y. Zhao, "The temperature effect on the methane and $\mathrm{CO} 2$ adsorption capacities of Illinois coal," Fuel, vol. 211, pp. 241-250, 2018.

[4] M. Gao, Z. Zhang, Y. Xiangang, Q. Liu, and H. Chen, "The location optimum and permeability-enhancing effect of a low-level shield rock roadway," Rock Mechanics and Rock Engineering, vol. 51, no. 9, pp. 2935-2948, 2018.

[5] R. Sakurovs, S. Day, S. Weir, and G. Duffy, "Temperature dependence of sorption of gases by coals and charcoals," International Journal of Coal Geology, vol. 73, no. 3-4, pp. 250-258, 2008. 
[6] M. Gao, S. Zhang, and J. Li, "Wang, H. 2019. Dynamic failure mechanism of coal and gas outbursts and response mechanism of support structure," Thermal Science, vol. 23, pp. 122-122, 2019.

[7] H. P. Xie, "Research framework and anticipated results of deep rock mechanics and mining theory," Advanced Engineering Sciences, vol. 49, no. 2, 2017.

[8] H. P. Xie, F. Gao, and Y. Ju, "Research and development of rock mechanics in deep group engineering," Chinese Journal of Rock Mechanics and Engineering, vol. 34, no. 11, pp. 21612178, 2015.

[9] H. Xie, F. Gao, Y. Ju, R. Zhang, M. Gao, and J. Deng, "Novel idea and disruptive technologies for the exploration and research of deep earth," Advanced Engineering Sciences, vol. 49 , no. $1,2017$.

[10] Z. Wen, S. Jing, Y. Jiang et al., "Study of the fracture law of overlying strata under water based on the flow-stress-damage model," Geofluids, vol. 2019, Article ID 3161852, 12 pages, 2019.

[11] H. P. Xie, M. Z. Gao, R. Zhang et al., "Study on concept and progress of in situ fidelity coring of deep rocks," Chinese Journal of Rock Mechanics and Engineering, vol. 39, no. 5, 2020.

[12] X. Wang, Z.-j. Wen, Y. Jiang, and H. Huang, "Experimental study on mechanical and acoustic emission characteristics of rock-like material under non-uniformly distributed loads," Rock Mechanics and Rock Engineering, vol. 51, no. 3, pp. 729-745, 2018.

[13] W. Zhijie, W. Xiao, Y. Tan, H. Zhang, W. Huang, and L. Qinghai, "A study of rockburst hazard evaluation method in coal mine," Shock and Vibration, vol. 2016, Article ID 8740868, 9 pages, 2016.

[14] Z. Hao, F. Yi-Xiong, G. Yi-Cong, and T. Jianrong, "The solving process of conceptual design for complex product based on performance evolution," Journal of Mechnal Enginnering, vol. 54, no. 9, 2018.

[15] W. Qing-Lin and X. Hui-Feng, "Virtual knowledge flow generation algorithm supporting complex product systems design," Computer Engineering and Applications, vol. 53, no. 22, pp. 29-34, 2017.

[16] W. Y. Dong, Z. T. Hua, L. Dan, M. Zhou, and Y. Q. Wang, "Data-driven multi-attribute optimization decision-making for complex product design schemes," China Mechanical Engineering, vol. 31, no. 7, 2020.

[17] S. Berrezzoug, A. Boudjemal, and F. T. Bendimerad, "Internative design and multidisciplinary optimization of geostationary communication satellite," International Journal on Interactive Design and Manufacturing, vol. 5, 2019.

[18] C. Greengalgh, Large-Scale Collaborative Virtual Environments. Department of Computer Science, Nottingham University, Nottingham, UK, 1997.

[19] T. V. R. M. L. Parisi, "Low tech illusion for the world wide web," in Digital Illusion, C. Dodsworth Jr., Ed., pp. 129-136, ACM press, New York, NY. USA, 1998.

[20] P. Zhang, H. Feng, T. D. Yang, B. Y. Zhao, J. G. Sun, and R. H. Tan, "Innovative design process model of TRIZ and digital twin integration iterative evolution based on parameter deduction," Computer Integrated Manufacturing Systems, vol. 25, no. $6,2019$.

[21] J. Guo, N. Zhao, L. Sun, and S. Zhang, "Modular based flexible digital twin for factory design," Journal of Ambient and Humanized Computing, vol. 7, 2018.
[22] S. Victor and K. E. Willcox, "Engineering design with digital thread," AIIA Journal, vol. 56, no. 11, pp. 4515-4528, 2018.

[23] B. Schleich, N. Anwer, L. Mathieu, and S. Wartzack, "Shaping the digital twin for design and production engineering," CIRP Annals, vol. 66, no. 1, pp. 141-144, 2017.

[24] C. B. Zhuang, J. H. Liu, H. Xiong, X. Ding, S. Liu, and G. Weng, "Connotation, architecture and trends of product digital twin," Computer Integrated Manufacturing Systems, vol. 23, no. 4, 2017.

[25] F. Tao, J. Cheng, Q. Qi, M. Zhang, H. Zhang, and F. Sui, "Digital twin-driven product design, manufacturing and service with big data," International Journal of Advanced Manufacturing Technology, vol. 94, no. 9-12, pp. 3563-3576, 2018.

[26] H. Li, F. Tao, Q. H. Wang et al., "Integration framework and key technology of complex product design-manufacturing based on digital twin," Computer Integrated Manufacturing Systems, vol. 25, no. 6, 2019.

[27] H. Wan, W. Zhao, X. Hua, B. Yu, X. Guo, and L. Chen, "Numerical study of influence of deep coring parameters on temperature of in-situ core," Thermal Science, vol. 23, no. 3, Part A, pp. 1441-1447, 2019.

[28] Y. Y. Song, F. Z. Cai, B. P. Zhang, B. H. Ye, E. W. Wei, and M. Y. Liang, "Study of information integration of production conceptual design and structure design," Journal of Tsinghua University, vol. 38, no. 2, 1998.

[29] X. Liu, S. Huang, Z. Q. Chen, and X. Luo, "Conceptual design based on TRIZ \& function analogy for product innovation," Journal of Mechanical Engineering, vol. 52, no. 23, p. 34, 2016.

[30] D. Y. Yang, Z. G. Xu, J. F. Zhu, K. Y. Su, and Z. Y. Zhu, "Design of dismantling equipment scheme based on axiomatic design and polychromatic stesComputer Integrated Manufacturing Systems," vol. 25, no. 10, 2019.

[31] S.-H. Jung, S.-J. Baek, and Y.-Y. Yu, “A study on applying TRIZ to logistics improvement," Journal of Digital Convergence, vol. 12, no. 8, pp. 77-84, 2014.

[32] W. H. Xia, K. Wang, Y. Li, and Y. Xiong, "Innovative design for adaptive module of in-pipe robot based on TRIZ," Journal of Mechanical Engineering, vol. 52, no. 5, 2016.

[33] X. Guo, J. Wang, W. Zhao, K. Zhang, and C. Wang, "Study of medical device innovation design strategy based on demand analysis and process case base," Multimedia Tools \& Applications, vol. 75, no. 22, pp. 14351-14365, 2016.

[34] T. Run-Hua, Triz and Application Technology Innovation Process and Method, Higher education press, 2010.

[35] T. Rashid, I. Beg, and S. M. Husnine, "Robot selection by using generalized interval-valued fuzzy numbers with TOPSIS," Applied Soft Computing, vol. 21, pp. 462-468, 2014.

[36] K. Yoon, Systems Selection by Multiple Attribute Decision Making, [Ph.D. thesis], Dissertation, Kansas State University, 1980.

[37] D. Streimikiene, T. Balezentis, I. Krisciukaitiene, and A. Balezentis, "Prioritizing sus-tainable electricity production technologies: MCDM approach," Renewable \& Sustainable Energy Reviews, vol. 16, no. 5, pp. 3302-3311, 2012.

[38] M. Zeleny, Linear Multiobjective Programming, Springer-Verlag, Berlin, 1974. 